\title{
A physical map of the papaya genome with integrated genetic map and genome sequence
} Qingyi Yu1 ${ }^{1}$ Eric Tong1, Rachel L Skelton ${ }^{1}$, John E Bowers ${ }^{2}$, Meghan R Jones ${ }^{1}$, Jan E Murray ${ }^{1,3}$, Shaobin Hou ${ }^{4}$, Peizhu Guan' ${ }^{5}$, Ricelle A Acob5 ${ }^{5}$, MingCheng Luo ${ }^{6}$, Paul H Moore ${ }^{7}$, Maqsudul Alam ${ }^{4}$, Andrew H Paterson ${ }^{2}$ and Ray Ming*1,3

Address: ${ }^{1}$ Cellular and Molecular Biology Research Unit, Hawaii Agriculture Research Center, Aiea, HI 96701, USA, ${ }^{2}$ Plant Genome Mapping Laboratory, University of Georgia, Athens, GA 30602, USA, ${ }^{3}$ Department of Plant Biology, University of Illinois at Urbana-Champaign, Urbana, IL 61801, USA, ${ }^{4}$ Center for Advanced Studies in Genomics, Proteomics and Bioinformatics, University of Hawaii, Honolulu, HI 96822, USA, ${ }^{5}$ Department of Molecular Bioscience and Bioengineering, University of Hawaii, Honolulu, HI 96822, USA, ${ }^{6}$ Department of Plant Sciences, University of California, Davis, CA 95616, USA and 7USDA-ARS, Pacific Basin Agricultural Research Center, Hilo, HI 96720, USA

Email: Qingyi Yu - qyu@ag.tamu.edu ; Eric Tong - etong@harc-hspa.com; Rachel L Skelton - rachelostroff@yahoo.com; John E Bowers - jebowera@uga.edu; Meghan R Jones - meghanrjones@gmail.com; Jan E Murray - jmurray@life.uiuc.edu; Shaobin Hou - shaobin@hawaii.edu; Peizhu Guan - peizhu@hawaii.edu; Ricelle A Acob - richelle@hawaii.edu; MingCheng Luo - mcluo@ucdavis.edu; Paul H Moore - PMoore@harc-hspa.com; Maqsudul Alam - alam@hawaii.edu;

Andrew H Paterson - paterson@dogwood.botany.uga.edu; Ray Ming* - rming@life.uiuc.edu

* Corresponding author

Published: 7 August 2009

BMC Genomics 2009, 10:37| doi:10.1186/|47|-2164-10-37|
Received: 4 February 2009

Accepted: 7 August 2009

This article is available from: http://www.biomedcentral.com//47/-2/64//0/37।

(c) 2009 Yu et al; licensee BioMed Central Ltd.

This is an Open Access article distributed under the terms of the Creative Commons Attribution License (http://creativecommons.org/licenses/by/2.0), which permits unrestricted use, distribution, and reproduction in any medium, provided the original work is properly cited.

\begin{abstract}
Background: Papaya is a major fruit crop in tropical and subtropical regions worldwide and has primitive sex chromosomes controlling sex determination in this trioecious species. The papaya genome was recently sequenced because of its agricultural importance, unique biological features, and successful application of transgenic papaya for resistance to papaya ringspot virus. As a part of the genome sequencing project, we constructed a BAC-based physical map using a high information-content fingerprinting approach to assist whole genome shotgun sequence assembly.

Results: The physical map consists of 963 contigs, representing $9.4 \times$ genome equivalents, and was integrated with the genetic map and genome sequence using BAC end sequences and a sequence-tagged high-density genetic map. The estimated genome coverage of the physical map is about $95.8 \%$, while $72.4 \%$ of the genome was aligned to the genetic map. A total of $\mathrm{I}, 18 \mathrm{I}$ high quality overgo (overlapping oligonucleotide) probes representing conserved sequences in Arabidopsis and genetically mapped loci in Brassica were anchored on the physical map, which provides a foundation for comparative genomics in the Brassicales. The integrated genetic and physical map aligned with the genome sequence revealed recombination hotspots as well as regions suppressed for recombination across the genome, particularly on the recently evolved sex chromosomes. Suppression of recombination spread to the adjacent region of the male specific region of the $Y$ chromosome (MSY), and recombination rates were recovered gradually and then exceeded the genome average. Recombination hotspots were observed at about $10 \mathrm{Mb}$ away on both sides of the MSY, showing 7-fold increase compared with the genome wide average, demonstrating the dynamics of recombination of the sex chromosomes.
\end{abstract}

Conclusion: A BAC-based physical map of papaya was constructed and integrated with the genetic map and genome sequence. The integrated map facilitated the draft genome assembly, and is a valuable resource for comparative genomics and map-based cloning of agronomically and economically important genes and for sex chromosome research. 


\section{Background}

Papaya, Carica papaya L., is a fast-growing fruit crop grown in tropical and subtropical regions worldwide. Papaya fruit is among the most nutritious available. It is rich in vitamins $\mathrm{A}$ and $\mathrm{C}$, and has been recommended for prevention of vitamin A deficiency in tropical and subtropical developing countries [1]. In addition to fresh fruit production, papaya is grown for papain, a proteolytic enzyme widely used in food processing, cosmetic, pharmaceutical, and leather industries, as well as medical applications $[2,3]$.

The majority of flowering plants, unlike most animal species that produce unisexual individuals, produce 'perfect' flowers that contain both male and female organs. It has been reported that only $6 \%$ of 250,000 angiosperm species are dioecious, i.e. having male and female individuals [4]. Papaya is one of the rare species classified as trioecious because its individuals exist as one of three sex types - female, male, or hermaphrodite. Sex determination in papaya is controlled by a pair of recently evolved sex chromosomes with the genotype XX for female, XY for male, and $X Y^{h}$ for hermaphrodite $[5,6]$. The difference between $\mathrm{X}$ and $\mathrm{Y}$ (or $\mathrm{Y}^{\mathrm{h}}$ ) chromosomes is a small non-recombination region, which is called the male-specific region of the $\mathrm{Y}$ chromosome (MSY). The $\mathrm{Y}$ and $\mathrm{Y}^{\mathrm{h}}$ originated from an ancestral Y chromosome about 73,000 years ago and share $98.6 \%$ DNA sequence identity in the MSY [7]. The physical size of the MSY is about $8 \mathrm{Mbp}$ and accounts for only about $13 \%$ of the papaya $\mathrm{Y}$ chromosome $[8,9]$. Papaya is an excellent system in which to study the early events of sex chromosome evolution.

Papaya originated in Central America where it was domesticated by aboriginals in this region [10]. Hermaphrodite papaya trees are self-pollinated and hermaphrodite cultivars are in production in most papaya growing regions for the obvious reason that every hermaphrodite tree produces fruit. Nevertheless, dioecious cultivars are used in India, Australia, and South Africa to assure greater fruit production under cool winter temperatures. The first transgenic papaya cultivar SunUp is derived from hermaphrodite Solo cultivar Sunset that has undergone more than 25 generations of self pollination [10]. The genomic DNA of hermaphrodite 'SunUp', that contains the X and $\mathrm{Y}^{\mathrm{h}}$ chromosomes, was used for construction of a papaya bacterial artificial chromosome (BAC) library [11], whereas a 'SunUp' female with XX chromosomes was used for whole genome shotgun sequencing to avoid the complication of assembling the heterozygous region of the $\mathrm{X}$ and $\mathrm{Yh}^{\mathrm{h}}$ chromosomes [9].

Papaya belongs to the small family Caricacea and is in the order Brassicales. Papaya and Arabidopsis diverged from a common ancestor about 72 million years ago. Thus, papaya can serve as an outgroup for comparative study of Brassicaceae genomes. Papaya has a small genome of 372 Mbp with nine pairs of chromosomes, a short juvenile phase of 3-8 months, and a short generation time of 9-15 months. Papaya is one of the few fruit tree crops that can flower and fruit throughout the year, providing a constant supply of flower buds and fruits. Each mature fruit contains 800-1000 seeds, and a single F1 tree could produce a large segregating F2 population for genetic studies. Clonal propagation can be done easily from cuttings or through micro-propagation. An efficient transformation system has been established as demonstrated by the success of transgenic papaya that saved the Hawaiian papaya industry [12,13]. These attributes make papaya an excellent model system for tropical fruit trees in which to study a number of biological processes, including sex chromosome evolution $[5,7,8]$, cell wall biosynthesis and degradation [14], vegetative/reproductive growth phase transition, flower development $[15,16]$, fruit development, fruit ripening, and post-harvesting physiology [14].

Significant progress has been made in recent years in developing genomic resources to expedite genome research in papaya. A BAC library of 'SunUp' hermaphrodite was constructed with $13.7 \times$ genome equivalents, providing the foundation for studying papaya genome structure and organization [11]. BAC ends of this library were sequenced [17], providing the first glimpse of the sequence composition of the papaya genome. Two highdensity genetic linkage maps have been constructed $[18,19]$, providing essential tools for comparative genomic analysis, marker-assisted selection, and genomic dissection of complex traits. The first high-density genetic map of papaya was constructed with 1,501 markers, including 1,498 amplified fragment length polymorphism (AFLP) markers, 2 morphological markers, and one transgenic marker [18]. Although this map has highdensity, it is not suitable for aligning papaya genome sequence to linkage groups and integrating genetic and physical maps due to the anonymous nature of AFLP markers. To overcome this limitation, highly informative sequence-based simple sequence repeat (SSR) markers were used to construct the second high-density genetic map, containing 707 markers including 706 sequencebased SSR markers and one morphological marker [19]. These SSR markers were developed from either BAC end or whole-genome shotgun sequence reads [19], and this map is a crucial resource for integration of genetic and physical maps and the genome sequences.

Several methods have been developed for construction of physical maps, including hybridization-based [20] and fingerprinting-based methods [21]. Fingerprinting techniques have been widely used in construction of genomewide physical maps. A number of techniques have been 
developed to generate fingerprints including the traditional agarose-gel, acrylamide gel, and automated capillary sequencer-based high-information-content fingerprinting methods [21-23]. After comparatively evaluating five fingerprinting methods, it was concluded that the high-information-content fingerprinting method with five enzyme digestion and SNaPshot labeling developed by Luo et al. [22] is the most effective [21]. We report here the construction of a BAC-based physical map of the papaya genome using high-information-content fingerprinting [22]. Contigs on the physical map were aligned with the papaya genome sequence assembly through BAC-end sequences (BES).

\section{Results}

\section{Fingerprinting and contig map assembly}

The papaya BAC library used for fingerprinting was constructed from hermaphrodite 'SunUp', the original transgenic cultivar [11]. This BAC library includes 39,168 BAC clones with the average insert size of $132 \mathrm{~kb}$, providing $13.7 \times$ papaya-genome equivalents. All 39,168 BAC clones were fingerprinted and the failed clones were repeated twice, obtaining successful fingerprints for 38,522 BAC clones $(98.4 \%$ of the BAC library). After excluding the clones with no or small inserts (referred as less than 20 true fragments after editing), and cross-contamination (70\% or higher shared fragments for neighboring clones), the remaining 30,824 fingerprints $(78.7 \%$ of the $\mathrm{BAC}$ library) were subjected to contig assembly using the FPC (FingerPrinted Contigs) program.

After automated overlap evaluation and manual review, 26,466 BAC clones were assembled into 963 contigs, while 4,358 clones remained as singletons. The average number of fragments (bands) of each clone was 69.4. Considering the clones with no or small inserts were excluded from contig assembly and the average insert size of the BAC clones used for contig assembly should be higher than $132 \mathrm{~kb}$, the BAC clones mapped on contigs $(26,466$ BAC clones) represented at least $9.4 \times$ genome equivalents of the papaya genome. A total of 571 contigs (59.3\% of contigs) contained more than 9 BAC clones with a total of 25,122 BAC clones, accounting for $81.5 \%$ of the mapped clones. On average, each contig contains 27.5 BAC clones. The longest contig contains 1571 consensus bands, which is about $0.7 \%$ of the total length of the FPC contigs http://www.stardaddy.uga.edu/fpc/ WebAGCoL/papaya/WebFPC/. A summary of the papaya FPC map is shown in Table 1.

\section{Overgo and single-copy probe hybridization}

A total of 2,277 overgo probes representing conserved sequences in Arabidopsis and genetically mapped Brassica loci were tested against 36,864 papaya BACs. A total of 1,329 overgos ( $58 \%$ of overgos designed) detected posi-
Table I: Summary of the papaya FPC physical map constructed by high information content fingerprinting

\begin{tabular}{lr}
\hline Number of clones with successful fingerprints & 30,824 \\
\hline Number of singletons & 4,358 \\
\hline Number of clones on contigs & 26,466 \\
\hline Number of contigs & 963 \\
\hline Contig size distribution & \\
\hline 200 & 3 \\
$100-199$ & 34 \\
$50-99$ & 155 \\
$25-49$ & 174 \\
$10-24$ & 205 \\
$3-9$ & 188 \\
2 clones & 204 \\
\hline
\end{tabular}

tive BACs in the papaya. After eliminating low quality data, the remaining 1,181 overgos were anchored on the papaya FPC map. Among these overgos, 756 (64.0\%) hit single contigs; the average number of clones per overgo is 6.0 .

Sixteen probes representing single-copy loci of papaya were used to screen the papaya BAC library. A total of 153 positive BAC clones were obtained. These 16 single-copy probes were anchored on the FPC map. Fifteen out of the 16 probes hit single contigs and only the probe of papaya pistillata (PI) gene hit two contigs, ctg1350 and ctg577. The ctg577 contains only three clones and all of them are PI positive clones. Thus, $\operatorname{ctg} 577$ should be part of the ctg1350, but the high stringency setting failed to merge them into a single contig. The probe of tetA gene from the transformation vector [12] was placed on $\operatorname{ctg} 972$. The ctg972 contains 347 BAC clones, while the total number of consensus bands (CB) is only 557 . The detailed analysis of ctg972 is shown in the Genome Coverage section. Most clones on ctg972 contain chloroplast sequences. We sequenced 4947 bp upstream and 1713 bp downstream of the flanking regions of tetA insertion and the result showed that the 4947 bp upstream fragment shared $98 \%$ identity with the papaya chloroplast genome [24]. We conclude that the tetA probe was placed on $\operatorname{ctg} 972$ due to the border sequence sharing high similarity with the chloroplast genome.

\section{$B A C$ end sequencing}

The entire papaya BAC library of 39,168 BAC clones was end-sequenced. After the first round sequencing, the failed clones were re-arrayed in 384-well plates and resequenced. A total of 67,179 BAC end sequences passed the filter after trimming the vector sequences and elimi- 
nating the clones with cross-well contamination. The total length of the trimmed sequences is $44,725,370 \mathrm{bp}$, which accounts for $12 \%$ of the papaya genome. The average length of each read is $665.76 \mathrm{bp}$ and the $\mathrm{G}+\mathrm{C}$ content of the BAC end sequences is $35.0 \%$. Paired ends from 32,397 BAC clones were used to build scaffolds for the wholegenome shotgun sequence assembly and provide anchor points for alignment of the FPC physical map with genome sequences and integration of the genetic and physical maps.

\section{Integration of physical map, genetic map, and genome sequence}

The sequence-tagged SSR genetic map of papaya was used for integrating the genetic and physical maps with the genome sequences [19]. Of the 706 mapped SSR markers, 153 were derived from BAC end sequences (BESs), 466 were designed from shotgun sequence reads, and 87 were developed from assembled shotgun contigs. The BESderived SSRs directly anchored the FPC contigs to the genetic map. From the 153 BES-derived microsatellites, 122 FPC contigs containing 46,475 consensus bands and 97 shotgun scaffolds covering $132 \mathrm{Mb}$ (35.5\% of the papaya genome) were anchored to the genetic map. The 32,397 paired BESs were aligned to the shotgun scaffolds to extend the integrated map. The remaining 553 mapped microsatellites were positioned on shotgun scaffolds by BlastN search. The BAC clones with paired ends covering the mapped microsatellites were used as baits to search the FPC physical map to find the corresponding FPC contigs. The order and orientation of the FPC contigs were examined by aligning on the shotgun scaffolds. The integrated map was further extended with information from the shotgun scaffolds. Additional BAC clones on the anchored shotgun scaffolds were used to search the FPC map for extending the integrated map.

The initial integration was conducted by searching the shotgun sequence using BESs of the BAC clones containing the mapped microsatellites to place the shotgun scaffolds and FPC contigs on the genetic map. Detailed information on the initial integration of linkage group 1 (LG1) is showed in Figure 1 and Additional file 1. Among the 77 mapped SSRs on LG1, four were not found in shotgun scaffolds, and three of the four SSRs were MSY-linked, which are not in the female genome. Three SSRs were found in the shotgun scaffolds, but no BAC containing these SSRs was found. Two FPC contigs that were placed on LG1 in the initial integrated map were also mapped onto other linkage groups and were thus removed from our final integrated map. We also used shotgun sequence scaffolds and FPC contigs to validate the initial integration. Overlapping scaffolds and FPC contigs confirmed their order on the genetic map (Additional files 1, 2 \&3).

\section{LG1}

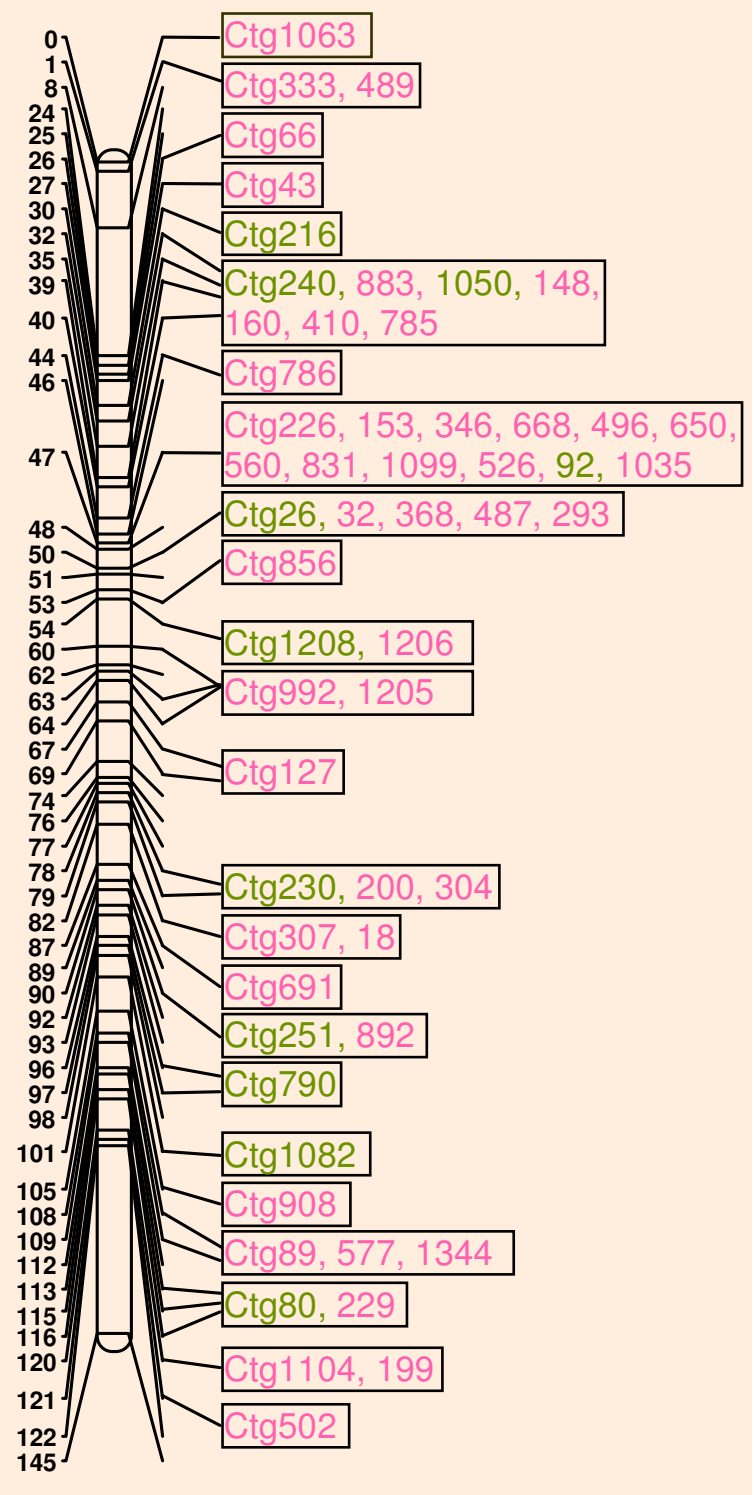

Figure I

The integration of FPC contigs and LG I of the papaya genome. The numerical scale at the left is the cumulative length of LGI in centimorgans. The FPC contigs in green color were mapped to LGI using BES-derived SSRs. The FPC contigs in pink color were mapped to LGI using shotgun sequence-derived SSRs. The three layers of information, genetic map, FPC contigs, and WGS scaffolds, can be found in the Additional files 2 \&3. 
For example, FPC contig 1050 connected scaffolds 14 and 140 on LG1. FPC contig 786 corrected the genetic location of the three scaffolds 99, 123, and 39. Eleven genetic loci that had been incorrectly mapped were identified and relocated. Two potential scaffold merges, involving scaffolds 794 and 215, and scaffolds 26 and 286, were recognized. The validated map is shown in Additional file 2. The initial erroneously mapped genetic markers might be the result of segregation distortion and the limited number of individuals used for mapping.

In the final integrated map, 535 (55.6\%) FPC contigs containing 21,371 (81.2\%) BAC clones and 168,217 consensus bands were anchored on the genetic linkage map. A total of 255 shotgun scaffolds covering $233 \mathrm{Mb}$ were anchored to the genetic map. Overall, 63\% of the papaya genome sequences were placed on the genetic map.

Both the FPC contigs and the shotgun scaffolds bridged the gap between linkage groups 8 and 10. Linkage groups 9 and 11 were merged based on fluorescence in situ hybridization (FISH) [9]. The estimated physical size of each linkage group is based on the length of each linkage group in centimorgans. Coverage of the integrated map on each linkage group is not even. Linkage group $9+11$ showed the highest coverage. Over $90 \%$ of this linkage group was covered by anchored shotgun scaffolds and almost $100 \%$ was covered by assigned FPC contigs. The least covered linkage group, LG1, is the sex chromosome for which $13 \%$ is heterologous male specific region, covering only $43.5 \%$ of the estimated physical size (Table 2 and Additional file 1).

\section{Genome coverage}

Lengths of the fingerprinted contigs are presented as consensus band (CB) units. To determine average band size and estimate the genome coverage of the fingerprinted physical map, we used information from the draft sequence of the papaya genome. Twenty-two non-overlapping fingerprinted contigs were aligned to the shotgun assembly through BESs (Additional file 4). These 22 FPC contigs of 7,503 CB units cover 12,256,987 bp. We determined that the average band size in our papaya FPC map is about $1.6 \mathrm{~kb}$.

We investigated the organelle genome contamination in our FPC physical map to better estimate genome coverage. Two DNA probes containing sorghum chloroplast ropB and $\operatorname{trn} K$ genes were used to screen the 'SunUp' hermaphrodite papaya BAC library and a total of 568 positive BAC clones were identified, with 179 identified by both probes. We searched for these 568 clones in the papaya

Table 2: Summary of the integrated genetic and physical map

\begin{tabular}{|c|c|c|c|c|c|c|c|c|c|c|c|}
\hline \multicolumn{4}{|c|}{ Genetic Map } & \multicolumn{3}{|c|}{ Shotgun Sequence } & \multicolumn{5}{|c|}{ Physical Map } \\
\hline $\begin{array}{l}\text { Linkage } \\
\text { Group }\end{array}$ & $\begin{array}{c}\text { No. } \\
\text { mapped } \\
\text { SSR } \\
\text { Markers }\end{array}$ & $\begin{array}{l}\text { Size } \\
(\mathrm{cM})\end{array}$ & $\begin{array}{l}\text { Estimated } \\
\text { Physical } \\
\text { Size (Mbp) }\end{array}$ & $\begin{array}{c}\text { No. } \\
\text { aligned } \\
\text { Scaffolds }\end{array}$ & $\begin{array}{l}\text { Length } \\
\text { (Mbp) }\end{array}$ & Coverage & $\begin{array}{l}\text { No. aligned } \\
\text { FPC Ctgs }\end{array}$ & $\begin{array}{l}\text { No. aligned } \\
\text { BACs }\end{array}$ & $\begin{array}{l}\text { Length } \\
\text { (CB units) }\end{array}$ & $\begin{array}{l}\text { Estimated } \\
\text { Physical } \\
\text { Size (Mbp) }\end{array}$ & Coverage \\
\hline LGI & 77 & 145.0 & 50.48 & 25 & 21.97 & $43.52 \%$ & 56 & 1892 & 15682 & 25.09 & $49.70 \%$ \\
\hline LG2 & 70 & 138.8 & 48.32 & 29 & 27.07 & $56.02 \%$ & 63 & 2345 & 18877 & 30.20 & $62.50 \%$ \\
\hline LG3 & 116 & 132.4 & 46.09 & 35 & 29.89 & $64.85 \%$ & 68 & 2796 & 22187 & 35.50 & $77.02 \%$ \\
\hline LG4 & 57 & 120.6 & 41.98 & 30 & 22.07 & $52.57 \%$ & 44 & 1840 & 13946 & 22.31 & $53.14 \%$ \\
\hline LG5 & 63 & 103.6 & 36.07 & 22 & 24.33 & $67.45 \%$ & 48 & 2287 & 17704 & 28.33 & $78.54 \%$ \\
\hline LG6 & 106 & 100.2 & 34.88 & 36 & 29.42 & $84.35 \%$ & 65 & 2819 & 21458 & 34.33 & $98.42 \%$ \\
\hline LG7 & 59 & 96.4 & 33.56 & 25 & 21.36 & $63.65 \%$ & 62 & 2050 & 17602 & 28.16 & $83.91 \%$ \\
\hline LG8+10 & 72 & 118.9 & 41.39 & 29 & 27.59 & $66.66 \%$ & 64 & 2502 & 19538 & 31.26 & $75.53 \%$ \\
\hline LG9+II & 81 & 91.3 & 31.78 & 22 & 28.70 & $90.31 \%$ & 63 & 2780 & 20770 & 33.23 & $104.56 \%$ \\
\hline LGI 2 & 5 & 21.4 & 7.45 & 2 & 0.96 & $12.89 \%$ & 2 & 60 & 453 & 0.72 & $9.66 \%$ \\
\hline Total & 706 & 1068.6 & 372 & 255 & 233.37 & $62.73 \%$ & 535 & 21371 & 168217 & 269.15 & $72.35 \%$ \\
\hline
\end{tabular}


FPC map and found 145 of these either formed singletons or were excluded from FPC map construction. Among the remaining 423 clones, 229 were placed on contig 972, 9 on contig 426, and 185 were distributed over 60 other contigs. The BAC end sequences of the 568 positive BAC clones were compared with the papaya chloroplast genome sequence [unpublished data] and $93 \mathrm{BAC}$ clones were identified as having both ends sharing over $90 \%$ identity with papaya chloroplast genome sequence. The insert sizes of these $93 \mathrm{BAC}$ clones were determined by Contour-clamped homogeneous electric field (CHEF) electrophoresis. Twelve were confirmed to contain the chloroplast genome sequence by comparing their real measured sizes with the sizes derived from in silico analysis of their BESs on the papaya chloroplast genome sequence. Ten out of the 12 BACs were placed on contig 972 and the other two BACs were excluded from the FPC map.

The papaya mitochondrial genome sequence was used as a query to search the papaya BES database. A total of 356 $\mathrm{BAC}$ clones was identified with at least one end sharing over $95 \%$ identity with the papaya mitochondrial genome sequence. Among them, 144 BACs were verified with both ends containing the papaya mitochondrial genome sequences. Among the 144 BACs, 57 either formed singletons or were excluded from FPC map construction, 26 were placed on contig 867,24 on contig 524,15 on contig 1172 , 4 on contig 47,4 on contig 553 , and the rest were distributed on 10 other contigs.

The total length of the papaya FPC map is 224,354 CB units and the average number of consensus bands of each contig is 233.0. To estimate the genome coverage of this map, we excluded contigs 972, 426, 867, 524, 1172, 47, and 553, which were confirmed to contain papaya organelle genome sequences. The total length of the remaining contigs was $222,808 \mathrm{CB}$ units and $356.5 \mathrm{Mb}$ $(95.8 \%)$ of the papaya genome was covered in these FPC contigs. Based on these estimates, we calculated that $269.15 \mathrm{Mb}$ (72.4\%) of the papaya genome was anchored to the genetic map (Table 2).

\section{Genetic recombination}

With the shotgun sequences and the FPC contigs both aligned on the genetic map, we examined the relationships between genetic distance and physical distance in the papaya genome. From the papaya genome size of 372 $\mathrm{Mb}$ and the total genetic map length of $1068.6 \mathrm{cM}$, we calculated that the average physical distance per centimorgan of the papaya genome is about $348 \mathrm{~kb}$. We aligned the FPC contigs on the genetic map (Additional file 5). On the integrated map, the ratio between genetic and physical distance varies among chromosomes. Recombination suppressed regions were observed near the center of all major linkage groups (Additional file 5). Hotspots of recombination were found on one end of linkage groups $2,3,4$, and 5 .

The longest linkage group, LG1, contains the MSY. We investigated the variation of the ratio between genetic and physical distance across LG1 (Figure 2). One region with severely suppressed recombination was shown on the center of LG1, and this region is the MSY. The recombination rates at the adjacent regions on both sides of the MSY were gradually recovered as the distance to the MSY increases; dramatically elevated at the locations about 10 $\mathrm{Mb}$ away from the MSY to 7-fold of the genome average; and then dropped again. Based on our integrated map, this region covers about $3.9 \mathrm{Mb}$, which is about $17 \%$ of the shotgun sequence aligned on LG1. Since the shotgun sequence aligned the genetic map covered only $43.5 \%$ of the entire LG1, we estimated the size of the suppression region to be about $8-9 \mathrm{Mb}$.

\section{Discussion}

The sequenced papaya SunUp genome is derived from inbred cultivar Sunset with three transgenic insertions, and the papaya genome has no recent genome wide duplication except the ancient triplication event shared by most eudicots $[9,25,26]$. The genomic organization of papaya is therefore less complicated than that of Arabidopsis, which has undergone two rounds of genome wide duplication; or the highly heterozygous poplar genome that has undergone one recent genome wide duplication. Nevertheless, the integration of a BAC-based physical map, genome sequence, and genetic map helped to correct errors and enhance the quality of these resources that are complementary to one another. Integration of these three sets of genomic information provides a valuable resource for comparative genomics, particularly for those researchers who work on the Brassicales [27].

\section{Genome coverage and representation}

The papaya genome size of $372 \mathrm{Mb}$ was estimated by flow cytometry [28]. The papaya draft genome sequence presented an opportunity to verify the genome size estimate by comparing the whole genome shotgun sequence to the fully assembled BAC sequences. However, the only fully sequenced papaya BACs are those on the MSY and its corresponding region of the $\mathrm{X}$ chromosome. Since the female papaya genome was sequenced, comparison with the Xspecific BACs is valid. Two fully sequenced X BACs, 53E18 and $61 \mathrm{H02}$, consist of 251,868 bp and 168,440 bp, respectively [8]. The whole genome shotgun (WGS) sequence matched $70.3 \%$ of the combined 420,308 bp BAC sequences so that the genome size of papaya could be $385 \mathrm{Mb}$ based on the assembled $271 \mathrm{Mb}$ WGS sequence [9]. However, the MSY of papaya is pericentromeric and even the $\mathrm{X}$ counterpart has more repetitive sequence than 


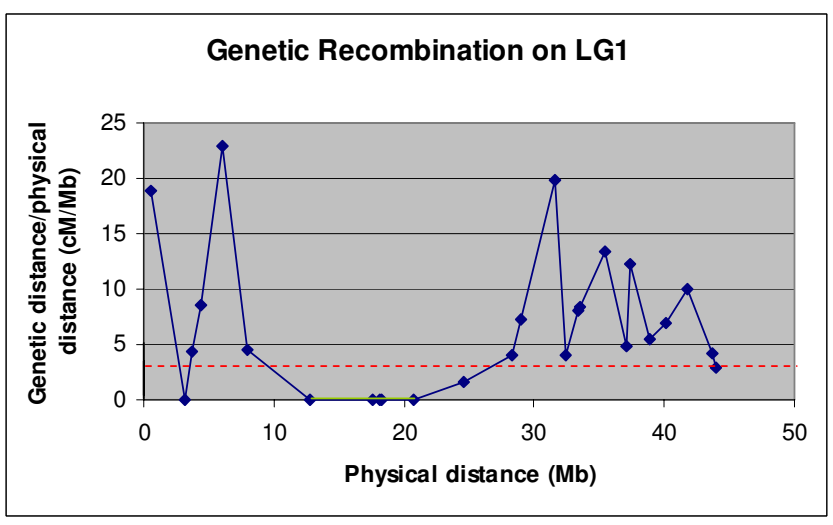

Figure 2

Variation of recombination rates on LGI of the papaya integrated map. The $X$ axis represents the physical distance of integrated contigs in megabases along LGI. The $Y$ axis represents the recombination rates per Mb DNA sequence $(\mathrm{cM} / \mathrm{Mb})$. The average rates of recombination across the genome and across the LGI are at $2.9 \mathrm{cM} / \mathrm{Mb}$ as indicated by the dashed red line. The green line highlighted the recombination rate of the MSY region.

the genome average $[29,30]$, thus the WGS sequence could have been underrepresented in this region. For this reason, the original estimate of $372 \mathrm{Mb}$ might be closer to the actual genome size of papaya.

The papaya physical map was based on the patterns of fragments generated by restriction enzyme digestion. We used the high stringency setting to build up the contig map to minimize false end-merges. Some ends of the contigs might overlap but failed to form larger contigs due to insufficient consensus bands for the high stringency of a minimum $70 \%$ overlapping bands. This could result in an overestimation of the genome coverage of the physical map. However, in our assembled FPC map, 4,358 or 14\% of clones remained as singletons and $18.5 \%$ of the clones located on the small contigs (containing fewer than 9 clones). Compared with the large contigs, singletons and small contigs may be more important for filling gaps in the physical map. Our estimation of the coverage of the papaya physical map was based on the consensus bands and the singletons were not included. Thus, while the genome coverage of the papaya physical map might be overestimated, the scale should be limited.

Accuracy of the genome coverage estimate is also related to the number of contigs in the physical map. The physical map of Populus consists of 2,802 contigs, representing $9.4 \times$ genome equivalents [31]. The total length of the estimated sizes of the FPC contigs is $577 \mathrm{Mb}$, about 20\% larger than the Populus genome size of $485 \mathrm{Mb}$ [31]. In addition to Populus, overestimation of genome coverage was also found in the soybean physical map [32]. The soybean physical map consists of 2,905 contigs due to the large number of chromosomes and its highly repetitive genome [32]. The total estimated length of contigs in the soybean physical map is $1,408 \mathrm{Mb}$, about $26.3 \%$ larger than the soybean genome size of $1,115 \mathrm{Mb}$ [32]. In contrast to the maps with large numbers of contigs, in Arabidopsis, 27 contigs covered the entire genome except for the centromere, telomere, and nucleolus organization regions (NOR) [20]. In rice, 458 contigs represent about $90.6 \%$ of the rice genome [33]. Papaya is a domesticated and inbreeding species and the estimated residual heterozygosity is only about $0.06 \%$ for the sequenced gynodioecious cultivar SunUp [9]. The physical map of the papaya genome consists of 963 contigs, which is about $1 / 3$ of the numbers of contigs in the Populus and soybean physical maps. Our estimate of the genome coverage of the papaya physical map might be close to the actual percentage.

The level of genome coverage of the physical map relies on the coverage of the underlying BAC library. The BAC library we used for construction of the papaya physical map included 39,168 BAC clones and provided 13.7x genome equivalents. A BAC library usually does not completely cover an entire genome due to non-random distribution of target restriction sites in certain regions of the genome. Moreover, highly repetitive fragments, such as centromeres, telomeres, and NOR regions, are under-represented in the BAC libraries. In papaya, the highly repetitive MSY is a good example of these difficult regions. Extreme gene paucity and a high density of retroelements were observed in the papaya MSY [31]. In our current physical map of the papaya MSY in a separate project to study the evolution of sex chromosomes, the SunUp hermaphrodite BAC library contains nearly all of the 8-9 Mb MSY region (R. Ming, P.H. Moore, A.H. Paterson, J. Jiang, and Q. Yu unpublished data). The physical map of the MSY indicates outstanding coverage of the papaya genome by this BAC library, considering that the centromere of the Y chromosome is likely within the MSY [33].

\section{Genetic recombination}

DAPI (4', 6-diamidino-2-phenylindole) stained papaya chromosomes revealed highly condensed heterochromatin knobs located in the centromeric and pericentromeric regions of all nine pairs of chromosomes [9]. Lengths of the condensed heterochromatin knobs varied among the chromosomes [9]. We examined the genome-wide recombination of papaya based on our integrated map. Consistent with papaya chromosome structure, recombination suppression regions were found at or near the center of all the major linkage groups, suggesting that those regions might be centromeric. It has been demonstrated that the rate of recombination varies among chromosomes, across individual chromosomes, and between different sex types 
[34-36]. Severe recombination suppression was found in the centromeric and pericentromeric heterochromatin of several species [37-41]. A 53-fold reduction in recombination frequency was found in the left pericentromere of the Arabidopsis centromere 1 (CEN1), a 10-fold reduction in the right pericentromere, and 200-fold reduction in the centromeric core [42]. A large quantity of repeats, duplications, and insertions were accumulated in the centromeric and pericentromeric regions, while very few genes were found in these regions $[43,44]$.

The MSY on LG1 is extensively suppressed for recombination. The estimated size of the suppressed region was about 8-9 Mb based on the integrated genetic and physical map, consistent with the 8-9 Mb physical map estimate of the male specific region of the Y chromosome [8]. The physical location of the MSY is in the middle of the Y chromosome based on pachytene FISH images [30], but the genetic map of the MSY was on the upper half of the MSY, likely due to the lower recombination rate on one arm of the Y chromosome [16]. Indeed, pachytene FISH images showed the denser heterochromatic regions on one of the chromosome arms [9]. Fewer markers mapped on this chromosome arm resulting in fewer anchored WGS sequences; it is possible that fewer WGS sequence could be assembled due to the repetitive nature of the heterochromatic regions on this arm. The location of the MSY in Figure 2 is likely distorted for this reason and the physical size of LG1 is underestimated. Interestingly, suppression of recombination spread to adjacent regions of the MSY, but recombination rates recovered gradually first and then increased dramatically at about $10 \mathrm{Mb}$ from the MSY resulting in a hotspot of $20 \mathrm{cM}$ per Mb. This is equivalent to $50 \mathrm{~kb}$ per $\mathrm{cM}$, a 7 -fold increase compared to the genome wide average of $348 \mathrm{~kb}$ per $\mathrm{cM}$. Beyond these two hotspots, the recombination rate declined in the remaining region of both arms with an average about $7.5 \mathrm{cM}$ per $\mathrm{Mb}$ at $133 \mathrm{~kb}$ per $\mathrm{cM}$ or 2.6-fold increase than the genome wide average. Our results demonstrated the dynamics of recombination in a pair of evolving young sex chromosomes that have changed since the initiation of the MSY, and are different from the sudden increase recombination in the small $2.6 \mathrm{Mb}$ and $400 \mathrm{~kb}$ pseudo-autosomal regions of the ancient human Y chromosome [45].

It is well known that recombination rate varies along chromosomes. Recombination rates are higher in telomeric regions when compared to centromeric and chromosomal regions. In the human genome, the mean intensity per hotspot is $0.115 \mathrm{cM}$ in telomeric regions, while the mean intensity is $0.070 \mathrm{cM}$ in centromeric regions [46]. Consistently, most recombination suppression regions of papaya were found in the middle of chromosomes and several recombination hotspots were found on one end of LGs 2, 3, 4, and 5. It has been reported that recombination rates were higher in regions with higher gene density [4648]. Recombination hotspots would be the regions favored by natural selection to produce beneficial allele combinations. To determine whether the recombination hotspots found in papaya are located in gene-riched regions, we would need fine-scale mapping of recombination rates across the genome.

\section{Potential applications}

A genetic linkage map is constructed by placing genetic loci on chromosomes based on recombination frequencies, while the physical map is constructed based on overlapping restriction patterns of large insert BAC clones. Integration of the genetic and physical maps revealed recombination hotspots as well as regions suppressed for recombination. The integrated genetic and physical map allows estimates of physical distances between genetic markers, and provided the framework for assembling the whole genome shotgun sequences. Meanwhile, assembled genome sequence provided precise physical distances between genes and DNA markers in gapless regions. These three genomic resources complement one another and correct errors from each individual source. The combined information enhances the capacity for map-based cloning and identification of underlying genes controlling quantitative traits in papaya.

The draft genome sequence contained $92 \%$ of the genes in papaya [9]. To uncover the genes controlling complex traits, we will need to rely on mapping quantitative trait loci (QTLs) and map-based cloning. The integrated map reported here will simplify the process of map-based cloning. A total of 706 SSR markers were integrated with the physical map. Using a subset of these mapped SSR markers to screen segregating populations will allow QTLs to be mapped on intervals of the genetic map, and fine mapping can be carried out using markers from BAC end sequences or WGS sequences within an interval. Most QTLs controlling economically important traits in papaya have not yet been characterized, including those for controlling sugar content, fruit size, shape, and weight, and some complex disease reactions. We expect that our integrated map and genome sequence will expedite the mapping and cloning of target genes and facilitate papaya breeding through marker-assisted selection.

The current draft sequence of the papaya genome represents about $75 \%$ of the papaya genome [9]. With rapid progress in next generation sequencing technology, additional papaya whole genome sequencing may be carried out in the foreseeable future. The papaya BAC-based physical map integrated with the genome sequences and genetic map will be an essential resource for closing gaps of any particular genomic region under investigation. In addition, this integrated resource can help identify hetero- 
chromatic regions that are difficult to sequence using the current WGS approach, so that a different strategy might be carried out to fill these gaps when effective methods become available. Moreover, the integrated genetic and physical map could also provide a framework to guide the genome sequencing of related species.

A total of 1,181 overgos representing conserved sequences of Arabidopsis and genetically mapped Brassica loci were anchored on the integrated genetic and physical map and the draft genome sequence of papaya. These overgos are direct links among papaya, Arabidopsis and Brassica genomes for comparative genomic research among species within the order Brassicales. The overgos were designed from single-copy genes and sequences of Arabidopsis and Brassica. These anchored overgos further improved the quality of the physical map construction. Along with the FPC contigs and WGS sequences, overgo markers could help identify synteny and rearrangements in target regions of these genomes, particularly in these recently duplicated genomes of Arabidopsis and Brassica [49-51].

Carica papaya is the only species of the genus Carica. The small gene pool has limited papaya improvement through traditional breeding. For example, the papaya ringspot virus (PRSV) severely damaged papaya production worldwide. However, development of PRSV-resistant papaya using traditional breeding approaches was not successful due to the lack of PRSV-resistant genes in papaya. Vasconcellea, a closely related sister genus of papaya, contains genes resistant to several major virus and fungus diseases, including PRSV. The integrated genetic and physical map can serve as a reference to study gene and genome evolution, to reveal the genetic base of unique ecological adaptations of Carica and Vasconcellea, and to clone the disease resistance genes from Vasconcellea species for papaya improvement.

\section{Conclusion}

We have constructed a BAC-based physical map of Carica papaya using high information-content fingerprinting and overgo hybridization of conserved DNA probes from Arabidopsis and Brassica. This physical map was integrated with a sequence tagged high-density genetic map and a draft genome sequence. The integrated map revealed recombination cold and hot spots in the papaya genome, often associated with known chromosomal features such as the sex chromosomes, centromeres, and telomeres. The recombination rates of the pericentromeric regions are reduced but not completely suppressed. The complete suppression of the 8-9 Mb MSY and the rise and fall of recombination rates in flanking regions indicated the recombination rates in this specialized region evolved over the course of sex chromosome evolution, particularly in these early stages. The integrated map with the draft genome sequence and anchored overgo probes from Arabidopsis and Brassica is a valuable resource for the plant research community.

\section{Methods \\ $B A C D N A$ isolation and fingerprinting}

BAC DNA was isolated with Qiagen R.E.A.L. Prep 96 Plasmid Kit (Qiagen, Valencia, CA) according to the manufacturer's instruction. The purified BAC DNA was fingerprinted according to Luo et al. [19]. BAC DNA was digested simultaneously with five restriction enzymes, BamHI, EcoRI, XbaI, XhoI, and HaeIII, and labeled with SNaPshot Multiplex System (Applied Biosystems, Foster City, CA). The labeled samples were purified and dissolved in $10 \mu \mathrm{l}$ of Hi-Di formamide with internal size standard LIZ-500 (Applied Biosystems, Foster City, CA) and labeled restriction fragments were sized with an $A B I$ 3700 DNA Analyzer (Applied Biosystems, Foster City, CA) using POP5 polymer. Fragment size-calling was performed with GeneMapper software (Applied Biosystems, Foster City, CA).

\section{Physical map construction}

Fingerprint profile of each clone collected by the ABI data collection program was processed with the combination of software packages GenoProfiler [52] and FPMiner http://www.bioinforsoft.com/ to remove vector fragments, high frequency fragments, and cross-contaminated clones. The edited sizes data were used for contig assembly with FPC V8.5.3 http://www.agcol.arizona.edu/soft ware/fpc/. Initial assembly was started at stringency of $1 \times$ $10^{-45}$ and a tolerance of $0.5 \mathrm{bp}$, followed by various steps of DQering, end-to-end merge, and manual securitization.

\section{Genetic map construction}

A mapping population of $54 \mathrm{~F}_{2}$ individuals derived from variety AU9 crossed by variety SunUp (pollen donor) was used for construction of a high-density genetic map [16]. A high-density genetic map was constructed using 707 markers, including 706 SSR markers and 1 morphological marker, fruit flesh color [16]. The resulting map consisted of 9 major and 3 minor linkage groups and spanned $1068.6 \mathrm{cM}$ with a density at $1.5 \mathrm{cM} /$ marker [16].

\section{Genome sequencing}

A total of 2.8 million whole-genome shotgun (WGS) sequencing reads were generated from variety SunUp by Sanger sequencers [9]. The plastid and mitochondrial genomes were assembled from whole-genome shotgun reads [9]. By using a combination of homology-based and de novo methods, a papaya repeat database was constructed [53-55]. After excluding organellar, repetitive, and low-quality reads, a total of 1.6 million reads were 
assembled into 47,483 contigs containing $271 \mathrm{Mb}$. With information from the integrated genetic and physical map and the BAC end sequences, a total of 17,764 scaffolds were assembled, covering $370 \mathrm{Mb}$ of the genome.

\section{BAC end sequencing}

Approximately 300 ng-400 ng BAC DNA of each clone was used for BAC end sequencing. The primers used were T7 5'-TAATACGACTCACTATAGGG and M13R 5'-GGATAACAATTTCACACAGG. The sequencing reaction was prepared using ABI BigDye Terminator v3.1 (Applied Biosystems, Foster City, CA) and then analyzed on ABI 3700 and ABI 3730xl DNA Analyzers (Applied Biosystems, Foster City, CA). The sequences were processed with PHRED and trimmed by LUCY. The trimmed BAC end sequences shorter than 100 bp were considered as failed ends and rearrayed into 384-well plates using Q-PIX (Genetix, Hampshire, UK) and re-sequenced.

\section{Overgo design and hybridization}

Overgo probes were designed using a Microsoft VISUAL BASIC program. Probes containing repetitive sequences were eliminated by comparison with The Institute for Genome Research (TIGR) Arabidopsis repeat database. These overgos were radioactively labeled and applied in multiplexed experiments consisting of 576 probes applied with triple redundancy $(24 \times 24 \times 24)$. Labeled filters were exposed to X-ray films for two weeks. Films were manually scored onto transparencies and scanned into a computer and read by ABBYY FINEREADER 5.0 software. Data were input into BACMan package http://plantge nome.agtec.uga.edu/bacman/ to deconvolute the multiplexed results into individual probes for BAC assignments.

\section{Screening BAC library}

High-density membranes of the papaya BAC library were pre-hybridized in $0.5 \mathrm{M} \mathrm{Na}_{2} \mathrm{HPO}_{4}, 7 \%$ SDS, $1 \mathrm{mM}$ EDTA, $100 \mathrm{ug} / \mathrm{ml}$ heat-denatured herring sperm DNA for at least four hours. Probes were labeled by random primer labeling system (RadiPrimerII, GE Healthcare Life Sciences, Piscataway, NJ). The hybridization was performed overnight in $0.5 \mathrm{M} \mathrm{Na} 2 \mathrm{HPO} 4,7 \% \mathrm{SDS}, 1 \mathrm{mM}$ EDTA, $100 \mathrm{ug} /$ $\mathrm{ml}$ heat-denatured herring sperm DNA with ${ }^{32} \mathrm{P}$ labeled probes at $65^{\circ} \mathrm{C}$. Hybridized membranes were washed twice in $0.5 \times \mathrm{SSPE}, 0.5 \% \mathrm{SDS}$ for $10 \mathrm{~min}$ at $65^{\circ} \mathrm{C}$. The washed membranes were exposed to $\mathrm{x}$-ray film with a pair of intensifying screens at $-80^{\circ} \mathrm{C}$ for $1-6$ days depending on the intensity of the signal.

\section{Mapping BAC end sequences to the draft genome}

BAC end sequence reads were trimmed by LUCY and screened for organellar sequences. BAC end sequences with both ends matching with organelle sequences were removed and the rest BAC end sequences were placed on the genome sequences by Arachne based on paired end assembling. The insert sizes of 18,700 BAC clones from the first ligation were estimated at $86 \mathrm{~Kb}$ and the rest 20,468 BAC clones from the second ligation contained inserts that averaged at $174 \mathrm{~Kb}$ [11].

\section{Authors' contributions}

QY and RM participated in conceiving and coordinating the study, analysis of data, and were the main authors responsible for writing the manuscript. QY, ET, RLS, MJ, JM, PG, and RAA carried out BAC DNA isolation. QY, ET, and RLS conducted BAC fingerprinting. JEB carried out Overgo hybridization. QY, ET, RLS, and SH participated in BES sequencing. QY and MCL participated in FPC contig assembling. PHM, MCL, AHP, and AM contributed to data analyses and interpretation of the results. All authors have read the manuscript and approved it.

\section{Additional material}

\author{
Additional file 1 \\ The detailed information of the initial integrated map of linkage \\ group 1. \\ Click here for file \\ [http://www.biomedcentral.com/content/supplementary/1471- \\ 2164-10-371-S1.xls]
}

\section{Additional file 2}

The detailed information of the final integrated map of linkage group 1.

Click here for file

[http://www.biomedcentral.com/content/supplementary/1471-

2164-10-371-S2.xls]

\section{Additional file 3}

The detailed information of the integrated genetic and physical map. Click here for file

[http://www.biomedcentral.com/content/supplementary/1471-

2164-10-371-S3.xls]

\section{Additional file 4}

Summary of the physical sizes of selected FPC contigs to estimate the average band size in FPC map.

Click here for file

[http://www.biomedcentral.com/content/supplementary/14712164-10-371-S4.doc]

\section{Additional file 5}

The FPC contigs were aligned on the genetic map. The numerical scale at the left of each linkage group is the cumulative length of the LG in centiMorgans. The pink boxes represent FPC contigs. The blue lines highlight the recombination suppression regions and the green lines highlight the recombination hotspots.

Click here for file

[http://www.biomedcentral.com/content/supplementary/14712164-10-371-S5.doc] 


\section{Acknowledgements}

We thank Farrell MacKenzie for technical assistance in BAC DNA isolation and fingerprinting and Ratnesh Singh for computer program support. This project was support by a US Department of Agriculture, Agricultural Research Services Cooperative Agreement (CA 58-3020-8-I34) with the Hawaii Agriculture Research Center.

\section{References}

I. Chandrika UG, Jansz ER, Wickramasinghe SMDN, Warnasuriya ND: Carotenoids in yellow- and red-fleshed papaya (Carica papaya L). J Sci Food Agric 2003, 83: I 279- 282.

2. Ockerman HW, Harnsawas S, Yetim H: Inhibition of papain in meat by potato protein or ascorbic acid. J Fod Sci 1993, 58: $1265-1268$.

3. Osato JA, Santiago L, Remo G, Cuadra M, Mori A: Antimicrobial and antioxidant activities of unripe papaya. Life Sci 1993, 53:1383-1389

4. Renner SS, Ricklefs RE: Dioecy and its correlates in the flowering plants. Am J Bot 1995, 82:596-606.

5. Liu Z, Moore PH, Ma H, Ackerman CM, Makandar R, Yu Q, Pearl HM, Kim MS, Charlton JW, Stiles JI, Zee FT, Paterson AH, Ming R: A primitive $Y$ chromosome in papaya marks incipient sex chromosome evolution. Nature 2004, 427:348-352.

6. Ming R, Yu Q, Moore PH: Sex determination in papaya. Seminars in Cell and Developmental Biology 2007, I 8:40I-408.

7. Yu Q, Navajas-Pérez R, Tong E, Robertson J, Moore PH, Paterson $\mathrm{AH}$, Ming R: Recent origin of dioecious and gynodioecious $\mathbf{Y}$ chromosomes in papaya. Topical Plant Biology 2008, I:49-57.

8. Yu Q, Hou S, Feltus FA, Jones MR, Murray J, Veatch O, Lemke C, Saw JH, Moore RC, Thimmapuram J, Liu L, Moore PH, Alam M, Jiang J, Paterson $A H$, Ming R: Low $X / Y$ divergence in four pairs of papaya sex-linked genes. Plant J 2008, 53:I24-I32.

9. Ming R, Hou S, Feng Y, Yu Q, Dionne-Laporte A, Saw JH, Senin P, Wang W, Ly BV, Lewis KL, Salzberg SL, Feng L, Jones MR, Skelton RL, Murray JE, Chen C, Qian W, Shen J, Du P, Eustice M, Tong E, Tang H, Lyons E, Paull RE, Michael TP, Wall K, Rice D, Albert H, Wang ML, Zhu YJ, Schatz M, Nagarajan N, Agbayani R, Guan P, Blas A, Wai CM, Ackerman CM, Ren Y, Liu C, Wang J, Wang J, Na JK, Shakirov EV, Haas B, Thimmapuram J, Nelson D, Wang X, Bowers JE, Gschwend AR, Delcher AL, Singh R, Suzuki JY, Tripathi S, Neupane K, Wei H, Irikura B, Paidi M, Jiang N, Zhang W, Presting G, Windsor A, NavajasPérez R, Torres MJ, Feltus Alex F, Porter B, Li Y, Burroughs AM, Luo MC, Liu L, Christopher DA, Mount SM, Moore PH, Sugimura T, Jiang J, Schuler MA, Friedman V, Mitchell-Olds T, Shippen DE, dePamphilis CW, Palmer JD, Freeling M, Paterson AH, Gonsalves D, Wang L, Alam M: The draft genome of the transgenic tropical fruit tree papaya (Carica papaya Linnaeus). Nature 2008, 452:991-996.

10. Storey WB: Papaya. In Outlines of Perennial Crop Breeding in the Tropics Edited by: Ferwerda FP, Wit FH. Wageningen: Veenman \& Zonen NV; 1969:389-408.

II. Ming R, Moore PH, Zee F, Abbey CA, Ma H, Paterson AH: Construction and characterization of a papaya BAC library as a foundation for molecular dissection of a tree-fruit genome. Theoretical and Applied Genetics 2001, I 02:892-899.

12. Fitch MM, Manshardt RM, Gonsalves D, Slightom JL: Virus resistance papaya derived from tissue bombarded with the coat protein gene of papaya ringspot virus. Bio/Tech 1992, 10:1466-|472.

13. Gonsalves D: Control of papaya ringspot virus in papaya: a case study. Annu Rev Phytopath 1998, 36:415-437.

14. Paull RE, Irikura B, Wu P, Turano H, Chen NJ, Blas A, Fellman JK, Gschwend AR, Wai CM, Yu Q, Presting G, Alam M, Ming R: Fruit development, ripening and quality related genes in the papaya genome. Tropical Plant Biology 2008, I:246-277.

15. Yu Q, Moore PH, Albert HH, Roader AH, Ming R: Cloning and characterization of a FLORICAULA/LEAFY ortholog, PFL, in polygamous papaya. Cell Research 2005, I 5:576-584.

16. Yu Q, Steiger D, Kramer E, Moore PH, Ming R: Floral MADS-Box genes in trioecious papaya: Characterization of $A G$ and $A P I$ subfamily genes revealed a sex-type-specific gene. Tropical Plant Biology 2008, I:97-107.

17. Lai CW, Yu Q, Hou S, Skelton RL, Jones MR, Lewis KL, Murray J, Eustice M, Guan P, Agbayani R, Moore PH, Ming R, Presting GG: Analy- sis of papaya BAC end sequences reveals first insights into the organization of a fruit tree genome. Mol Genet Genomics 2006, 276: $1-12$.

18. Ma H, Moore PH, Liu Z, Kim MS, Yu Q, Fitch MM, Sekioka T, Paterson $\mathrm{AH}$, Ming R: High-density linkage mapping revealed suppression of recombination at the sex determination locus in papaya. Genetics 2004, I 66:419-436.

19. Chen C, Yu Q, Hou S, Li Y, Eustice M, Skelton RL, Veatch O, Herds R, Diebold L, Saw J, Feng Y, Bynum L, Wang L, Moore PH, Paull RE, Alam $M$, Ming R: Construction of a sequence-tagged high-density genetic map of papaya for comparative structural and evolutionary genomics in brassicales. Genetics 2007, I 77:248I-249I.

20. Mozo T, Dewar K, Dunn P, Ecker JR, Fischer S, Kloska S, Lehrach H, Marra M, Martienssen R, Meier-Ewert S, Altmann T: A complete BAC-based physical map of the Arabidopsis thaliana genome. Nat Genet 1999, 22:271-275.

21. Nelson WM, Dvorak J, Luo M-C, Messing J, Wing RA, Soderlund C: Efficacy of clone fingerprinting methodologies. Genomics 2007, 89: $160-165$.

22. Luo MC, Thomas C, You FM, Hsiao J, Ouyang S, Buell CR, Malandro M, McGuire PE, Anderson OD, Dvorak J: High-throughput fingerprinting of bacterial artificial chromosomes using the SNaP. shot labeling kit and sizing of restriction fragments by capillary electrophoresis. Genomics 2003, 82:378-389.

23. Xu Z, Berg MA van den, Scheuring C, Covaleda L, Lu H, Santos FA, Uhm T, Lee MK, Wu C, Liu S, Zhang HB: Genome physical mapping from large-insert clones by fingerprint analysis with capillary electrophoresis: a robust physical map of Penicillium chrysogenum. Nucleic Acids Res 2005, 33:e50.

24. Suzuki JY, Tripathi S, Fermín GA, Jan F-J, Hou S, Saw H, Ackerman CM, Yu Q, Schatz MC, Pitz KY, Yépes M, Fitch MMM, Manshardt RM, Slightom JL, Ferreira SA, Salzberg SL, Alam M, Ming R, Moore PH, Gonsalves D: Characterization of insertion sites in Rainbow papaya, the first commercialized transgenic fruit crop. Tropical Plant Biology 2008, I:293-309.

25. Tang $H$, Wang $X$, Bowers JE, Ming $R$, Alam $M$, Paterson $A H$ : Unraveling ancient hexaploidy through multiply-aligned angiosperm gene maps. Genome Res 2008, I 8: I 944- 1954.

26. Tang H, Bowers JE, Wang X, Ming R, Alam M, Paterson AH: Synteny and colinearity in plant genomes. Science 2008, 320:486-488.

27. Freeling M, Lyons E, Pedersen B, Alam M, Ming R, Lisch D: Many or most genes in Arabidopsis transposed after the origin of the order Brassicales. Genome Res 2008, I 8: I924-1937.

28. Arumuganathan K, Earle ED: Nuclear DNA content of some important plant species. Plant Mol Biol Rep I991, 9:208-218.

29. Yu Q, Hou S, Hobza R, Feltus FA, Wang X, Jin W, Skelton RL, Blas A, Lemke C, Saw JH, Moore PH, Alam M, Jiang J, Paterson AH, Vyskot B, Ming R: Chromosomal location and gene paucity of the male specific region on papaya $\mathbf{Y}$ chromosome. Molecular Genetics and Genomics 2007, 278: I77-I85.

30. Zhang $W$, Wang $X, Y u$ Q, Ming R, Jiang J: DNA methylation and heterochromatinization in the male-specific region of the primitive $\mathbf{Y}$ chromosome of papaya. Genome Res 2008, I 8: 1938-1943.

31. Kelleher CT, Chiu R, Shin H, Bosdet IE, Krzywinski MI, Fjell CD, Wilkin J, Yin T, DiFazio SP, Ali J, Asano JK, Chan S, Cloutier A, Girn $\mathrm{N}$, Leach S, Lee D, Mathewson CA, Olson T, O'connor K, Prabhu AL, Smailus DE, Stott JM, Tsai M, Wye NH, Yang GS, Zhuang J, Holt RA, Putnam NH, Vrebalov J, Giovannoni JJ, Grimwood J, Schmutz J, Rokhsar D, Jones SJ, Marra MA, Tuskan GA, Bohlmann J, Ellis BE, Ritland K, Douglas CJ, Schein JE: A physical map of the highly heterozygous Populus genome: integration with the genome sequence and genetic map and analysis of haplotype variation. Plant J 2007, 50: 1063-1078.

32. Wu C, Sun S, Nimmakayala P, Santos FA, Meksem K, Springman R, Ding K, Lightfoot DA, Zhang HB: A BAC- and BIBAC-based physical map of the soybean genome. Genome Res 2004, 14:319-326.

33. Chen M, Presting G, Barbazuk WB, Goicoechea JL, Blackmon B, Fang G, Kim H, Frisch D, Yu Y, Sun S, Higingbottom S, Phimpgilai J, Phimphilai D, Thurmond S, Gaudette B, Li P, Liu J, Hatfield J, Main D, Farrar K, Henderson C, Barnett L, Costa R, Williams B, Walser S, Atkins M, Hall C, Budiman MA, Tomkins JP, Luo M, Bancroft I, Salse J, Regad F, Mohapatra T, Singh NK, Tyaqi AK, Soderlund C, Dean RA, Wing RA: 
An integrated physical and genetic map of the rice genome. Plant Cell 2002, I 4:537-545.

34. Coop G, Wen X, Ober C, Pritchard JK, Przeworski M: High-resolution mapping of crossovers reveals extensive variation in fine-scale recombination patterns among humans. Science 2008, 3 19:1395-1398.

35. Kong A, Gudbjartsson DF, Sainz J, Jonsdottir GM, Gudjonsson SA, Richardsson B, Sigurdardottir S, Barnard J, Hallbeck B, Masson G, Shlien A, Palsson ST, Frigge ML, Thorgeirsson TE, Gulcher JR, Stefansson $\mathrm{K}$ : A high-resolution recombination map of the human genome. Nature Genetics 2002, 31:241-247.

36. Kong A, Thorleifsson G, Stefansson H, Masson G, Helgason A, Gudbjartsson DF, Jonsdottir GM, Gudjonsson SA, Sverrisson S, Thorlacius T, Jonasdottir A, Hardarson GA, Palsson ST, Frigge ML, Gulcher $J R$, Thorsteinsdottir $U$, Stefansson K: Sequence variants in the RNF2I 2 gene associate with genome-wide recombination rate. Science 2008, 319:1398-1401.

37. Cook KR, Karpen GH: A rosy future for heterochromatin. Proc Natl Acad Sci USA 1994, 9 1:5219-5221.

38. Henikoff S: Conspiracy of silence among repeated transgenes. Bioessays 1998, 20:532-535.

39. Dimitri P, Junakovic N: Revising the selfish DNA hypothesis: new evidence on accumulation of transposable elements in heterochromatin. Trends Genet 1999, 15:123-124.

40. McCombie WR, de la Bastide M, Habermann K, Parnell LD, Dedhia N, Gnoj L, Schutz K, Huang E, Spiegel L, Yordan C, Sehkon M, Murray J, Sheet P, Cordes M, Threideh J, Stoneking T, Kalicki J, Graves T, Harmon G, Edwards J, Latreille P, Courtney L, Cloud J, Abbott A, Scott K, Johnson D, Minx P, Bentley D, Fulton B, Miller N, Greco T, Kemp K, Kramer J, Fulton L, Mardis E, Dante M, Pepin K, Hillier L, Nelson J, Spieth J, Simorowski J, May B, Ma P, Preston R, Vil D, See LH, Shekher M, Matero A, Shah R, Swaby I, O'Shaughnessy A, Rodriguez M, Hoffman J, Till S, Granat S, Shohdy N, Hasegawa A, Hameed A, Lodhi M, Johnson A, Chen E, Marra M, Wilson RK, Martienssen R: The complete sequence of a heterochromatic island from a higher eukaryote. Cell 2000, 100:377-386.

4I. Bowers JE, Arias MA, Asher R, Avise JA, Ball RT, Brewer GA, Buss RW, Chen AH, Edwards TM, Estill JC, Exum HE, Goff VH, Herrick KL, Steele CL, Karunakaran S, Lafayette GK, Lemke C, Marler BS, Masters SL, McMillan JM, Nelson LK, Newsome GA, Nwakanma CC, Odeh RN, Phelps CA, Rarick EA, Rogers CJ, Ryan SP, Slaughter KA, Soderlund $\mathrm{CA}$, Tang $\mathrm{H}$, Wing RA, Paterson $\mathrm{AH}$ : Comparative physical mapping links conservation of microsynteny to chromosome structure and recombination in grasses. Proc Natl Acad Sci USA 2005, 102:13206-13211.

42. Haupt W, Fischer TC, Winderl S, Fransz P, Torres-Ruiz RA: The CENTROMERE I (CENI) region of Arabidopsis thaliana: architecture and functional impact of chromatin. Plant J 200 I, 27:285-296.

43. Topp CN, Dawe RK: Reinterpreting pericentromeric heterochromatin. Current Opinion in Plant Biology 2006, 9:647-653.

44. Wang Y, Tang X, Cheng Z, Mueller L, Giovannoni J, Tanksley SD: Euchromatin and pericentromeric heterochromatin: comparative composition in the tomato genome. Genetics 2006, 172:2529-2540.

45. Henke A, Fischer C, Rappold GA: Genetic map of the human pseudoautosomal region reveals a high rate of recombination in female meiosis at the Xp telomere. Genomics 1993, 1 8:478-485

46. Myers S, Bottolo L, Freeman C, McVean G, Donnelly P: A fine-scale map of recombination rates and hotspots across the human genome. Science 2005, 310:321-324.

47. Fullerton SM, Bernardo Carvalho A, Clark AG: Local rates of recombination are positively correlated with GC content in the human genome. Mol Biol Evol 200 I, I 8: I I39-I I 42.

48. Kong A, Gudbjartsson DF, Sainz J, Jonsdottir GM, Gudjonsson SA, Richardsson B, Sigurdardottir S, Barnard J, Hallbeck B, Masson G, Shlien A, Palsson ST, Frigge ML, Thorgeirsson TE, Gulcher JR, Stefansson $\mathrm{K}$ : A high-resolution recombination map of the human genome. Nat Genet 2002, 3 I:24I-247.

49. Freeling M, Lyon E, Pedersen B, Alam M, Ming R, Lisch D: Many or most genes in Arabidopsis transposed after the origin of the order Brassicales. Genome Research 2008, 18:1924-1937.

50. Tang $H$, Wang $X$, Bowers JE, Ming R, Alam M, Paterson AH: Unraveling ancient hexaploidy through multiply aligned angiosperm gene maps. Genome Research 2008, 18:1944-1954.
5I. Lyons E, Pedersen B, Kane J, Freeling M: The value of nonmodel genomes and an example using synMap within CoGe to dissect the hexaploidy that predates the Rosids. Tropical Plant Biology 2008, I: I8I-I90.

52. You FM, Luo MC, Gu YQ, Lazo GR, Deal K, Dvorak J, Anderson OD: GenoProfiler: batch processing of high throughput capillary fingerprinting data. Bioinformatics 2007, 23:240-242.

53. Nagarajan N, Navajas-Pérez R, Pop M, Alam M, Ming R, Paterson AH, Salzberg SL: Genome-wide analysis of repetitive elements in papaya. Tropical Plant Biology 2008, I:I9|-20I.

54. Wang J, Chen C, Na JK, Yu Q, Hou S, Paull RE, Moore PH, Alam M, Ming R: Genome-wide comparative analysis of microsatellites in papaya. Tropical Plant Biology 2008, I:278-292.

55. Shakirov EV, Salzberg SL, Alam M, Shippen DE: Analysis of Carica papaya telomeres and telomere-associated proteins: Insights into the evolution of telomere maintenance in Brassicales. Tropical Plant Biology 2008, I:202-2 15.
Publish with Bio Med Central and every scientist can read your work free of charge

"BioMed Central will be the most significant development for disseminating the results of biomedical research in our lifetime. "

Sir Paul Nurse, Cancer Research UK

Your research papers will be:

- available free of charge to the entire biomedical community

- peer reviewed and published immediately upon acceptance

- cited in PubMed and archived on PubMed Central

- yours - you keep the copyright

Submit your manuscript here:

http://www.biomedcentral.com/info/publishing_adv.asp
BiolMedcentral 